\title{
A Note on Some Joint Distribution Functions involving the Time of Ruin
}

\author{
David C M Dickson \\ Centre for Actuarial Studies, Department of Economics, \\ University of Melbourne, VIC 3010, Australia \\ dcmd@unimelb.edu.au
}

\begin{abstract}
In a recent paper, Willmot (2015) derived an expression for the joint distribution function of the time of ruin and the deficit at ruin in the classical risk model. We show how his approach can be applied to obtain a simpler expression, and by interpreting this expression by probabilistic reasoning we obtain solutions for more general risk models. We also discuss how some of Willmot's results relate to existing literature on the probability and severity of ruin.
\end{abstract}

Keywords: Time of ruin; deficit at ruin; joint distribution function; MAP risk model

\section{Introduction}

This note is concerned with finite time ruin problems and its starting point is a recent paper by Willmot (2015) who considers the classical risk model. Our results extend beyond the classical risk model, but we start here with a description of this model. As we make many references to Willmot (2015), we follow his notation. Thus, the insurer's surplus process is $\left\{U_{t} ; t \geq 0\right\}$ where for $t \geq 0, U_{t}=u+c t-S_{t}$. Here $u \geq 0$ is the insurer's initial surplus, $c$ is the rate of premium income per unit time (assumed to be received continuously), and $S_{t}=\sum_{i=1}^{N_{t}} Y_{i}$ denotes the aggregate claim amount up to time $t$, where $\left\{N_{t} ; t \geq 0\right\}$ is a Poisson process with parameter $\lambda$ and $\left\{Y_{i}\right\}_{i=1}^{\infty}$ is a sequence of independent and identically distributed random variables, with $Y_{i}$ representing the amount of the $i$ th claim. Let $P(y)=\operatorname{Pr}\left(Y_{1} \leq y\right)=$ $1-\bar{P}(y), y \geq 0$, and let $p(y)=\frac{d}{d y} P(y)$. We assume that $c=(1+\theta) \lambda E\left(Y_{1}\right)$ where $\theta>0$ is the premium loading factor.

The time of ruin is denoted $T$ and is defined as $T=\inf \left\{t: U_{t}<0\right\}$ with $T=\infty$ if $U_{t} \geq 0$ for all $t \geq 0$. Further let $\left|U_{T}\right|$ denote the deficit at the time of ruin and let $U_{T^{-}}$denote the surplus immediately prior to ruin. We define the ultimate ruin probability as $\psi(u)=\operatorname{Pr}\left(T<\infty \mid U_{0}=u\right)=1-\phi(u)$, the 
finite time ruin probability as $\psi(u, t)=\operatorname{Pr}\left(T \leq t \mid U_{0}=u\right)=1-\phi(u, t)$, and what we call the $t$-deferred ruin probability as

$$
\bar{\psi}(u, t)=\operatorname{Pr}\left(t<T<\infty \mid U_{0}=u\right)=\psi(u)-\psi(u, t) .
$$

Similarly, we define probability and severity of ruin functions in finite and infinite time as $G(u, y)=\operatorname{Pr}\left(T<\infty,\left|U_{T}\right| \leq y \mid U_{0}=u\right)$ and $G(u, y, t)=$ $\operatorname{Pr}\left(T \leq t,\left|U_{T}\right| \leq y \mid U_{0}=u\right)$, with the $t$-deferred probability and severity of ruin function being

$$
\bar{G}(u, y, t)=\operatorname{Pr}\left(t<T<\infty,\left|U_{T}\right| \leq y \mid U_{0}=u\right)=G(u, y)-G(u, y, t) .
$$

Let $F(y, t)=\operatorname{Pr}\left(S_{t} \leq y\right)$, with density function $f(y, t)$ for $y>0$. Further, $F(0, t)=\operatorname{Pr}\left(N_{t}=0\right)=e^{-\lambda t}$.

Willmot (2015) considers the equation

$$
\frac{\partial}{\partial t} h(u, t)=c \frac{\partial}{\partial u} h(u, t)-\lambda h(u, t)+\lambda \int_{0}^{u} h(u-x, t) p(x) d x+\tau(u, t),
$$

and the special case when $\tau(u, t)=\tau(u)$. He solves these equations using Laplace transform techniques, applying different approaches for the special case and the general case, and finds solutions for $\phi(u, t)$ and $G(u, y, t)$ using the special case solution. The solution for $\phi(u, t)$ is the well-known Prabhu's (1961) formula (as expected!), and the formula for $G(u, y, t)$ is new. However, this new formula is somewhat complicated, and it is not easy to give a probabilistic interpretation of it.

Equations similar to the special case of (1.1) (i.e. $\tau(u, t)=\tau(u)$ ) have a long history in the risk theory literature; see Arfwedson (1950), Prabhu (1961) and references therein. In this literature $\tau(u)$ has a specific form that relates to $h(u, t)$ having a particular ruin-theoretic interpretation. Problems previously studied include $h(u, t)=\bar{\psi}(u, t)$, and this points to an alternative approach to finding a formula for $G(u, y, t)$, and to extensions, e.g. involving $U_{T^{-}}$. The solution we obtain for $G(u, y, t)$ in the next section has a clear probabilistic interpretation which indicates how we can obtain solutions for more general risk models.

The main contributions in this note are a simple formula for $\bar{G}(u, y, t)$ and an extension in the next section, and in Section 3 a generalisation of this formula for $\bar{G}(u, y, t)$ to the MAP risk model. In Section 4 we consider an alternative formula for $G(u, y, t)$.

\section{Joint distribution functions in the classical risk model}

Willmot (2015) applies the result in his Theorem 2 to obtain expressions for $\phi(u, t)$ and $G(u, y, t)$. We now apply the same result to obtain $\psi(u, t)$. This 
will illustrate our earlier point about a complicated formula (since $\psi(u, t)=$ $\left.\lim _{y \rightarrow \infty} G(u, y, t)\right)$, and will also serve to show that studying the $t$-deferred ruin probability provides a more straightforward approach to the problem of finding $\psi(u, t)$ and related probabilities from equation (1.1). Willmot's formula (17) yields the unsurprising result

$$
\psi(0, t)=\int_{0}^{t} w(0, s) d s
$$

where $w(0, t)=\frac{d}{d t} \psi(0, t)$ is the (defective) density of $T$ when $u=0$ (see Dickson and Willmot (2005) or Dickson (2007)), and for $u>0$ we obtain

$$
\begin{aligned}
\psi(u, t)= & e^{-\lambda t} \alpha(u+c t)-\alpha(u)+\int_{0}^{u+c t} \alpha(u+c t-x) f(x, t) d x \\
& -c \int_{0}^{t} \psi(0, s) f(u+c(t-s), t-s) d s
\end{aligned}
$$

where

$$
\alpha(u)=\frac{1}{\lambda \theta E\left(Y_{1}\right)} \int_{0}^{u} \phi(u-x) \lambda \bar{P}(x) d x .
$$

It is well known (e.g. Gerber (1979)) that

$$
\phi(u)=\phi(0)+\frac{\lambda}{c} \int_{0}^{u} \phi(u-x) \bar{P}(x) d x,
$$

giving

$$
\alpha(u)=\frac{c}{\lambda \theta E\left(Y_{1}\right)}(\phi(u)-\phi(0))=\frac{\phi(u)}{\phi(0)}-1
$$

since $\phi(0)=\theta /(1+\theta)$. Thus, by equation (2.1) we obtain

$$
\begin{aligned}
\psi(u, t)= & e^{-\lambda t}\left(\frac{\phi(u+c t)}{\phi(0)}-1\right)-\left(\frac{\phi(u)}{\phi(0)}-1\right)+\int_{0}^{u+c t}\left(\frac{\phi(u+c t-x)}{\phi(0)}-1\right) f(x, t) d x \\
& -c \int_{0}^{t} \psi(0, s) f(u+c(t-s), t-s) d s .
\end{aligned}
$$

This is a complicated formula, particularly when compared with writing $\psi(u, t)$ as the complement of Prabhu's formula for $\phi(u, t)$ (e.g. equation (5) in Willmot (2015)), nor does it appear to have a probabilistic interpretation. However, we can see from Prabhu's formula that

$\psi(u, t)=1-e^{-\lambda t}-\int_{0}^{u+c t} f(x, t) d x+c \int_{0}^{t} f(u+c s, s) d s-c \int_{0}^{t} f(u+c s, s) \psi(0, t-s) d s$.

Equating these two expressions for $\psi(u, t)$ we obtain

$$
\phi(u)=e^{-\lambda t} \phi(u+c t)+\int_{0}^{u+c t} \phi(u+c t-x) f(x, t) d x-c \int_{0}^{t} f(u+c s, s) \phi(0) d s,
$$


which does have a clear probabilistic interpretation. Although this is not a useful formula for finding $\phi(u)$, it is useful in our subsequent development.

We now obtain a formula for $\bar{\psi}(u, t)$. The objective in obtaining this formula is not to provide a means of calculating $\bar{\psi}(u, t)$, since the most efficient approach to this is to deduct $\phi(u)$ from Prabhu's formula as

$$
\bar{\psi}(u, t)=\psi(u)-\psi(u, t)=\phi(u, t)-\phi(u) .
$$

Rather, we seek a formula with a clear probabilistic interpretation that points the way to solutions to other problems. We see from equation (2.2) and Prabhu's formula that

$$
\begin{aligned}
\bar{\psi}(u, t)= & e^{-\lambda t} \psi(u+c t)+\int_{0}^{u+c t} \psi(u+c t-x) f(x, t) d x \\
& -c \int_{0}^{t} \bar{\psi}(0, t-s) f(u+c s, s) d s
\end{aligned}
$$

with $\bar{\psi}(0, t)=\psi(0)-\psi(0, t)$. Equation (2.3) has a simple interpretation the first two terms allow for ruin to occur from time $t$ with the surplus at time $t$ being $u+c t-x$, where $0 \leq x<u+c t$ is the aggregate claim amount at time $t$, and the final term adjusts for realisations of the surplus process that have caused ruin to occur before time $t$, using the same arguments as in Prabhu's formula.

Building on this interpretation, it follows that

$$
\begin{aligned}
\bar{G}(u, y, t)= & e^{-\lambda t} G(u+c t, y)+\int_{0}^{u+c t} G(u+c t-x, y) f(x, t) d x \\
& -c \int_{0}^{t} \bar{G}(0, y, t-s) f(u+c s, s) d s .
\end{aligned}
$$

This allows us to find $G(u, y, t)$ since the function $G(u, y)$ is well-documented (e.g. Gerber et al. (1987) and Drekic et al. (2004)), and we can find $G(0, y, t)$ easily, for example from equation (24) of Willmot (2015) which is just

$$
G(0, y, t)=\int_{0}^{t} \int_{0}^{y} w(0, x, s) d x d s
$$

where $w(0, x, s)$ is the (defective) joint density of the deficit at ruin $(x)$ and the time of ruin $(s)$ when $u=0$, a formula for which is given in Dickson (2007).

We make two observations about formula (2.4). First, it seems a much simpler formula to apply to obtain $G(u, y, t)$ (as $G(u, y)-\bar{G}(u, y, t)$ ) than formula (22) of Willmot (2015). In particular, the first two terms of (2.4) are simpler than the first two terms of Willmot's (22), whilst the third terms in (2.4) and Willmot's (22) are very similar. If our objective is simply to obtain the joint distribution function of $T$ and $\left|U_{T}\right|$ then (2.4) is a useful formula. 
However, it does not seem like a useful formula to obtain the joint density of $T$ and $\left|U_{T}\right|$ (nor does formula (22) of Willmot (2015)); in particular it is not obvious to the author how to obtain the expression for the joint density of $T$ and $\left|U_{T}\right|$ given in Dickson (2007) by differentiating (2.4).

Extending the previous interpretation, and now including the surplus prior to ruin, if we define

$$
H(u, z, y)=\operatorname{Pr}\left(T<\infty, U_{T^{-}} \leq z,\left|U_{T}\right| \leq y \mid U_{0}=u\right),
$$

and the $t$-deferred function

$$
\bar{H}(u, z, y, t)=\operatorname{Pr}\left(t<T<\infty, U_{T^{-}} \leq z,\left|U_{T}\right| \leq y \mid U_{0}=u\right),
$$

then we see that

$$
\begin{aligned}
\bar{H}(u, z, y, t)= & e^{-\lambda t} H(u+c t, z, y)+\int_{0}^{u+c t} H(u+c t-x, z, y) f(x, t) d x \\
& -c \int_{0}^{t} \bar{H}(0, z, y, t-s) f(u+c s, s) d s
\end{aligned}
$$

with $\bar{H}(0, z, y, t)=H(0, z, y)-H(0, z, y, t)$. In principle, we can evaluate $\bar{H}(0, z, y, t)$ by integrating the joint density of $U_{T^{-}},\left|U_{T}\right|$ and $T$ given by Dickson (2007) over the appropriate intervals, although this may not be a straightforward task depending on the choice of $P$.

The reason why the $t$-deferred functions (which include $\phi(u, t)=\operatorname{Pr}(T>$ $t)$ ) are easier to deal with is simple. Consider the equation satisfied by $\bar{H}(u, z, y, t)$ :

$\frac{\partial}{\partial t} \bar{H}(u, z, y, t)=c \frac{\partial}{\partial u} \bar{H}(u, z, y, t)-\lambda H(u, z, y, t)+\lambda \int_{0}^{u} \bar{H}(u-x, z, y, t) p(x) d x$,

so that $\tau(u, t)=0$ in equation (1.1), and hence the solution to equation (1.1) (from Willmot's Theorem 2) becomes

$$
\begin{aligned}
h(u, t)= & e^{-\lambda t} h(u+c t, 0)+\int_{0}^{u+c t} h(u+c t-x, 0) f(x, t) d x \\
& -c \int_{0}^{t} h(0, t-s) f(u+c s, s) d s .
\end{aligned}
$$

The key point with the $t$-deferred functions is that when we construct partial integro-differential equations for them by the method of infinitesimals, as we require ruin after time $t$, there is no term relating to ruin occurring in the initial time interval $(0, d t)$.

This also shows that if we can write $h(u, t)=h(u)-\bar{h}(u, t)$ where $\bar{h}(u, t)$ satisfies equation (1.1) with $\tau(u, t)=0$, then $h(u, t)$ must satisfy equation (1.1) with $\tau(u, t)=\tau(u)$. (Just replace $h(u, t)$ by $h(u)-h(u, t)$ in equation 
(1.1) to see this.) So, if $h(u, t)$ is defined in terms of the event $\{T \leq t\}$, then it is the special case of (1.1) that applies, i.e. $\tau(u, t)=\tau(u)$. An interesting open question is what sort of functions $h(u, t)$ satisfy equation (1.1) with $\tau(u, t)$ being a non-trivial function of $t$.

We now consider the special case, introduced by Willmot (2007), when $p(x+y)=\sum_{j=1}^{\infty} \kappa_{j}(x) \tau_{j}(y)$ for some functions $\left\{\kappa_{j}\right\}_{j=1}^{\infty}$ and density functions $\left\{\tau_{j}\right\}_{j=1}^{\infty}$. Starting from the joint density of $T$ and $\left|U_{T}\right|$ given in Dickson (2007) we can easily show that

$$
G(u, y, t)=\sum_{j=1}^{\infty} A_{j}(u, t) T_{j}(y)
$$

where $T_{j}(y)=\int_{0}^{y} \tau_{j}(x) d x, j=1,2,3, \ldots$ and

$$
A_{j}(0, t)=\lambda \int_{0}^{t}\left(e^{-\lambda s} \kappa_{j}(c s)+\int_{0}^{c s} \frac{x}{c s} f(c s-x, s) \kappa_{j}(x) d x\right) d s,
$$

and for $u>0$,

$$
\begin{aligned}
A_{j}(u, t)= & \lambda \int_{0}^{t}\left(e^{-\lambda s} \kappa_{j}(u+c s)+\int_{0}^{u+c s} f(x, s) \kappa_{j}(u+c s-x) d x\right) d s \\
& -c \int_{0}^{t} A_{j}(0, t-s) f(u+c s, s) d s .
\end{aligned}
$$

Further, if we define $A_{j}(u)=\lim _{t \rightarrow \infty} A_{j}(u, t)$, then $G(u, y)=\sum_{j=1}^{\infty} A_{j}(u) T_{j}(y)$. By formula (2.4), a similar decomposition holds for $\bar{G}(u, y, t)$ as

$$
\bar{G}(u, y, t)=\sum_{j=1}^{\infty} \bar{A}_{j}(u, t) T_{j}(y),
$$

where $\bar{A}_{j}(0, t)=A_{j}(0)-A_{j}(0, t)$ and for $u>0$

$$
\begin{aligned}
\bar{A}_{j}(u, t)= & e^{-\lambda t} A_{j}(u+c t)+\int_{0}^{u+c t} f(x, t) A_{j}(u+c t-x) d x \\
& -c \int_{0}^{t} \bar{A}_{j}(0, t-s) f(u+c s, s) d s .
\end{aligned}
$$

Calculation of $\bar{A}_{j}(u, t)$ appears to be more straightforward than calculation of $A_{j}(u, t)$. In particular, for many individual claim amount distributions, the function $A_{j}(\cdot)$ exists in a closed form making the calculation (by numerical integration) of the first line of formula (2.6) simpler than the calculation of the first line of formula (2.5).

We conclude this section by commenting on implementation of formula (2.4) and by giving two illustrations of the application of our formulae. It is not easy to obtain explicit expressions for $G(u, y, t)$ (or $\psi(u, t))$ because there 
are few individual claim amount distributions for which $f(x, t)$ exists in a closed form. In addition to the expression derived by Willmot (2015) in the case of mixed Erlang individual claims, explicit results are known for either $G(u, y, t)$ or the corresponding joint density in the case of Erlang claims (see Dickson (2007)) and in the case of Erlang(2) claims (a different formula) and claims distributed as a mixture of two exponential distributions (see Dickson (2008)). The common feature that all these formula have is that they are expressed in terms of infinite sums, and in the author's experience it requires considerable care to correctly program these formulae to overcome issues like overflow and underflow. From a programming point of view, the algorithm of Dickson and Waters (1992) to calculate approximations to $G(u, y, t)$ is much simpler to apply, and it produces very accurate results (see also Dickson (2008)).

For some claim size distributions, e.g. Erlang, all the components of formula (2.4) can be found, and accurate calculation of $G(u, y, t)$ is possible by numerical integration. At the very least, to apply formula (2.4) we require a known form for $G(u, y)$ and to be able to accurately calculate $f(x, t)$. If we have these we can then compute $\bar{G}(0, y, \tau)$ for $\tau<t$ by setting $u=0$ in formula (2.4), and then we can use these values with numerical integration to apply formula (2.4) with $u>0$. Similar comments apply to the application of Willmot's formula (22), although in this case we need a known form for $\psi(u)$ rather than $G(u, y)$ together with $f(x, t)$; this distinction matters little as we can find $G(u, y)$ for most claim size distributions for which $\psi(u)$ is known.

The one case for which we can very easily obtain an expression for $\bar{G}(u, y, t)$ from formula $(2.4)$ is when $P(x)=1-e^{-\alpha x}, x \geq 0$. In this case it is well-known (see, e.g., Gerber (1979)) that $w(0, y, t)=w(0, t) \alpha e^{-\alpha y}$. Then

$$
\bar{G}(0, y, t)=\int_{t}^{\infty} \int_{0}^{y} w(0, x, s) d x d s=\bar{\psi}(0, t)\left(1-e^{-\alpha y}\right) .
$$

Further, as $G(u, y)=\psi(u)\left(1-e^{-\alpha y}\right)$ (see, e.g., Gerber et al. (1987)), formula (2.4) becomes

$$
\begin{aligned}
\bar{G}(u, y, t)= & \left(e^{-\lambda t} \psi(u+c t)+\int_{0}^{u+c t} \psi(u+c t-x) f(x, t) d x\right. \\
& \left.-c \int_{0}^{t} \bar{\psi}(0, t-s) f(u+c s, s) d s\right)\left(1-e^{-\alpha y}\right),
\end{aligned}
$$

and from formula (2.3) it follows that

$$
\bar{G}(u, y, t)=\bar{\psi}(u, t)\left(1-e^{-\alpha y}\right) .
$$

The formula for $\psi(u)$ is well-known (see, e.g., Gerber (1979)) and, as noted by Willmot (2015), differing formulae for $\psi(u, t)$ exist in the literature and so a formula for $\bar{G}(u, y, t)$ can be found. 
We now illustrate the use of formula (2.6) by considering the $\operatorname{Erlang}(2, \beta)$ individual claims case with $p(x)=\beta^{2} x e^{-\beta x}, x>0$. For this case it is known that

$$
G(u, y)=A_{1}(u)\left(1-e^{-\beta y}\right)+A_{2}(u)\left(1-e^{-\beta y}(1+\beta y)\right),
$$

where for $i=1,2$

$$
A_{i}(u)=C_{i, 1} e^{-R_{1} u}+C_{i, 2} e^{-R_{2} u}
$$

where $0<R_{1}<\beta<R_{2}$ are the solutions to Lundberg's equation $\lambda+c r=$ $\lambda(1-r / \beta)^{-2}$ and $\left\{C_{i, j}\right\}$ can be found from Gerber et al. (1987) for $i, j=1,2$.

As $p(x+y)$ can be written as a combination of $\operatorname{Erlang}(1, \beta)$ and $\operatorname{Erlang}(2, \beta)$ density functions, we can apply formula (2.6). Let $\bar{A}_{1}(u, t)$ be the multiplier for the Erlang $(1, \beta)$ distribution function. Then the first two terms of $\bar{A}_{1}(u, t)$ become

$$
\begin{aligned}
& e^{-\lambda t} A_{1}(u+c t)+\int_{0}^{u+c t} f(x, t) A_{1}(u+c t-x) d x \\
= & \sum_{j=1}^{2} C_{1, j} e^{-R_{j}(u+c t)}\left(e^{-\lambda t}+\int_{0}^{u+c t} e^{R_{j} x} f(x, t) d x\right) \\
= & \sum_{j=1}^{2} C_{1, j} e^{-R_{j}(u+c t)}\left(e^{-\lambda t}+\sum_{n=1}^{\infty} e^{-\lambda t} \frac{(\lambda t)^{n}}{n !} \int_{0}^{u+c t} e^{R_{j} x} \frac{\beta^{2 n} x^{2 n-1} e^{-\beta x}}{\Gamma(2 n)} d x\right) \\
= & \sum_{j=1}^{2} C_{1, j} e^{-R_{j}(u+c t)} \sum_{n=0}^{\infty} e^{-\lambda t} \frac{(\lambda t)^{n}}{n !}\left(\frac{\beta}{\beta-R_{j}}\right)^{2 n}\left(1-\sum_{m=0}^{2 n-1} e^{-\left(\beta-R_{j}\right)(u+c t)} \frac{\left(\beta-R_{j}\right)^{m}(u+c t)^{m}}{m !}\right)
\end{aligned}
$$

with the usual convention that $\sum_{a}^{b}=0$ if $a>b$.

To obtain the third term of $\overline{A_{1}}(u, t)$ we use the result from Dickson (2007) that

$$
w(0, y, t)=\eta_{1}(0, t) \beta e^{-\beta y}+\eta_{2}(0, t) \beta^{2} y e^{-\beta y}
$$

where

$$
\eta_{1}(0, t)=2 \lambda \beta c t e^{-(\lambda+c \beta) t} \sum_{j=0}^{\infty} \frac{\left(\lambda \beta^{2} c^{2} t^{3}\right)^{j}}{j !(2 j+2) !}
$$

and

$$
\eta_{2}(0, t)=\lambda e^{-(\lambda+c \beta) t} \sum_{j=0}^{\infty} \frac{\left(\lambda \beta^{2} c^{2} t^{3}\right)^{j}}{j !(2 j+1) !} .
$$

Then

$$
\begin{aligned}
\bar{A}_{1}(0, t) & =\int_{t}^{\infty} \eta_{1}(0, r) d r=2 \lambda \beta c \sum_{j=0}^{\infty} \frac{\left(\lambda \beta^{2} c^{2}\right)^{j}}{j !(2 j+2) !} \int_{t}^{\infty} e^{-(\lambda+c \beta) r} r^{3 j+1} d r \\
& =2 \lambda \beta c \sum_{j=0}^{\infty} \frac{\left(\lambda \beta^{2} c^{2}\right)^{j}}{j !(2 j+2) !} \frac{\Gamma(3 j+2)}{(\lambda+c \beta)^{3 j+2}} e^{-(\lambda+c \beta) t} \sum_{k=0}^{3 j+1} \frac{((\lambda+c \beta) t)^{k}}{k !}
\end{aligned}
$$


which, for brevity, we write as

$$
\bar{A}_{1}(0, t)=\sum_{j=0}^{\infty} B_{j} e^{-(\lambda+c \beta) t} \sum_{k=0}^{3 j+1} \frac{((\lambda+c \beta) t)^{k}}{k !},
$$

where

$$
B_{j}=2 \lambda \beta c \frac{\left(\lambda \beta^{2} c^{2}\right)^{j}}{j !(2 j+2) !} \frac{\Gamma(3 j+2)}{(\lambda+c \beta)^{3 j+2}} .
$$

Then the integral in the third term of (2.6) becomes

$$
\begin{aligned}
& \int_{0}^{t} \sum_{j=0}^{\infty} B_{j} e^{-(\lambda+c \beta)(t-s)} \sum_{k=0}^{3 j+1} \frac{((\lambda+c \beta)(t-s))^{k}}{k !} \sum_{n=1}^{\infty} e^{-\lambda s} \frac{(\lambda s)^{n}}{n !} \frac{\beta^{2 n}(u+c s)^{2 n-1} e^{-\beta(u+c s)}}{\Gamma(2 n)} d s \\
= & e^{-\beta u-(\lambda+\beta c) t} \sum_{j=0}^{\infty} B_{j} \sum_{k=0}^{3 j+1} \frac{(\lambda+c \beta)^{k}}{k !} \sum_{n=1}^{\infty} \frac{\left(\lambda \beta^{2}\right)^{n}}{n ! \Gamma(2 n)} \int_{0}^{t}(t-s)^{k} s^{n}(u+c s)^{2 n-1} d s .
\end{aligned}
$$

Using a binomial expansion of $(u+c s)^{n-1}$ we can show that

$\int_{0}^{t}(t-s)^{k} s^{n}(u+c s)^{2 n-1} d s=\sum_{i=0}^{2 n-1}\left(\begin{array}{c}2 n-1 \\ i\end{array}\right) u^{2 n-1-i} c^{i} t^{n+i+k+1} \frac{(n+i) ! k !}{(n+k+i+1) !}$

which means we have a formula for all the components of $\bar{A}_{1}(u, t)$ and hence for $\bar{A}_{1}(u, t)$ itself. The same approach yields a similar formula for $\bar{A}_{2}(u, t)$.

Our approach leads to a different type of formula compared with existing results in that it is expressed in terms of the solutions of Lundberg's equation. As a contrast, see, for example, Garcia (2005) who provides a formula for $\phi(u, t)$ when the individual claim amount distribution is $\operatorname{Erlang}(2, \beta)$.

\section{Generalisation to the MAP risk model}

In this section we restrict our attention to the joint distribution of $T$ and $\left|U_{T}\right|$ which in the author's view is the most important joint distribution involving $T$.

We now consider a MAP risk model (e.g. Badescu et al. (2005), Cheung and Landriault (2009)). This is a general model which includes the classical risk model, the Markov-modulated risk model and the Sparre Andersen risk model with phase-type inter-claim times as special cases. In this risk model, the insurer's surplus is driven by an irreducible continuous-time Markov chain $\left\{J_{t} ; t>0\right\}$ on a state space $E=\{1,2, \ldots, m\}$. The premium income can vary according to the state, but the previous arguments apply only if the premium rate is a constant, $c$, independent of the state, so this is the case we consider. 
It is unnecessary to give a complete specification of the MAP risk model for the problem we consider, so we will not do so. A full description can be found in the references in the previous paragraph. Letting $U_{t}, N_{t}$ and $S_{t}$ denote surplus level, number of claims and aggregate claim amount at time $t$ for this model, we define

$$
q_{i, j}^{(n)}(t)=\operatorname{Pr}\left(N_{t}=n, J_{t}=j \mid J_{0}=i\right)
$$

for $i, j \in E$ and $n=0,1,2, \ldots$, to be the probability that, starting in state $i$, there are $n$ claims in $(0, t)$ and the state at time $t$ is $j$,

$$
F_{i, j}(x, t)=\operatorname{Pr}\left(S_{t} \leq x, J_{t}=j \mid J_{0}=i\right)
$$

for $i, j \in E$ and $x, t \geq 0$ to the probability that, starting in state $i$, the aggregate claim amount at time $t$ is at most $x$ and $J_{t}=j$, with $f_{i, j}(x, t)=$ $\frac{\partial}{\partial x} F_{i, j}(x, t)$, and

$$
\bar{G}_{i}(u, y, t)=\operatorname{Pr}\left(t<T<\infty,\left|U_{T}\right| \leq y \mid U_{0}=u, J_{0}=i\right)
$$

with $G_{i}(u, y)=\bar{G}_{i}(u, y, 0)$.

Using almost exactly the same interpretation that we used to write down formula (2.4) we have

$$
\begin{aligned}
\bar{G}_{i}(u, y, t)= & \sum_{j=1}^{m}\left(q_{i, j}^{(0)}(t) G_{j}(u+c t, y)+\int_{0}^{u+c t} f_{i, j}(x, t) G_{j}(u+c t-x, y) d x\right) \\
& -c \sum_{j=1}^{m} \int_{0}^{t} f_{i, j}(u+c s, s) \bar{G}_{j}(0, y, t-s) d s .
\end{aligned}
$$

The only change from the interpretation of formula (2.4) is that we must now allow for the state changing from $i$ at time 0 to $j \in E$ at time $t$ in the first two terms, and similarly for the state to have changed from $i$ at time 0 to $j \in E$ at the time of the last upcrossing of the the surplus process through 0 in the third term.

Li et al. (2015) provide a formula for the joint density of $T$ and $\left|U_{T}\right|$ for the MAP risk model with constant premium rate, from which the joint distribution function can be obtained, but formula (3.1) seems easier to implement, largely because it involves single integrals. However, it is by no means easy to apply formula (3.1). We must first set $u=0$ in formula (3.1) for $i=1,2, \ldots, m$, and solve the resulting equations numerically for $\bar{G}_{i}(0, y, t)$ in order to subsequently evaluate the integral term when $u>0$. A second issue with formula (3.1) is the calculation of densities $f_{i, j}$, particularly when trying to write a program to calculate the terms in the second integral. In general, a numerical approach is necessary, e.g. numerical inversion of the Laplace transform of $S_{t}$ provided by Ren (2008). One case where 
implementation appears relatively straightforward is the Sparre Andersen risk model with Erlang arrivals (assuming we can compute convolutions of the individual claim amount density).

We will not dwell on implementation of formula (3.1). The key point is that it is not difficult to extend formula (2.4). By contrast, it does not seem straightforward to extend Willmot's formula (22) to other risk models.

\section{Alternative formulae}

Returning to the classical risk model, Willmot's (2015) formula for $G(u, y, t)$ is based on the result in his Theorem 2. If we instead apply his Theorem 3 then we see that his formulae (49) and (50) for the case $u=0$ and his formula (48) for the case $u>0$ yield the unsurprising result that

$$
G(u, y, t)=\int_{0}^{t} \int_{0}^{y} w(u, x, s) d x d s
$$

for $u \geq 0$, where formulae for $w(u, x, s)$, the joint density of $\left|U_{T}\right|$ and $T$, can be found in Dickson (2007) - formula (7) for the case $u=0$ and formula (9) for the case $u>0$.

Now define

$$
\hat{G}(u, y, t)=\operatorname{Pr}\left(T \leq t,\left|U_{T}\right|>y \mid U_{0}=u\right)
$$

so that

$$
\hat{G}(u, y, t)=\psi(u, t)-G(u, y, t)=\int_{0}^{t} \int_{y}^{\infty} w(u, x, s) d x d s .
$$

Evaluating equation (4.1) we obtain for $u>0$

$$
\begin{aligned}
\hat{G}(u, y, t)= & \lambda \int_{0}^{t} e^{-\lambda s} \bar{P}(u+c s+y) d s \\
& +\lambda \int_{0}^{t} \int_{0}^{u+c s} f(x, s) \bar{P}(u+c s+y-x) d x d s \\
& -c \int_{0}^{t} f(u+c s, s) \hat{G}(0, y, t-s) d s,
\end{aligned}
$$

with

$\hat{G}(0, y, t)=\lambda \int_{0}^{t} e^{-\lambda s} \bar{P}(c s+y) d s+\lambda \int_{0}^{t} \int_{0}^{c s} \frac{x}{c s} f(c s-x, s) \bar{P}(x+y) d x d s$.

We remark that equation (4.3) is effectively the same as equation (24) in Willmot (2015). 
Equation (4.2) seems to yield a slightly simpler formula for $G(u, y, t$ ) (as $\hat{G}(u, 0, t)-\hat{G}(u, y, t))$ than equation (22) of Willmot (2015). An advantage of starting from Willmot's Theorem 3 rather than Theorem 2 is that the resulting expression for $G(u, y, t)$ is easily differentiated to obtain the joint density of $T$ and $\left|U_{T}\right|$. However, if computation of $G(u, y, t)$ is all that is required, formula (2.4) seems superior.

\section{References}

[1] Arfwedson, G. (1950) Some problems in the collective risk theory. Scandinavian Actuarial Journal, 1-38.

[2] Badescu, A., Breuer, L., Da Silva Soares, A., Latouche, G., Remiche, M.-A. and Standford, D. (2005) Risk processes analyzed as fluid queues. Scandinavian Actuarial Journal 2005, 127-141.

[3] Cheung, E.C.K. and Landriault, D. (2009) Perturbed MAP risk models with dividend barrier strategies. Journal of Applied Probability 46, 521541.

[4] Dickson, D.C.M. (2007) Some finite time ruin problems. Annals of Actuarial Science 2, 217-232.

[5] Dickson, D.C.M. (2008) Some explicit solutions for the joint density of the time of ruin and the deficit at ruin. ASTIN Bulletin 38, 259-279.

[6] Dickson, D.C.M. and Waters, H.R. (1992) The probability and severity of ruin in finite and in infinite time. ASTIN Bulletin 22, 177-190.

[7] Dickson, D.C.M. and Willmot, G.E. (2005) The density of the time to ruin in the classical Poisson risk model. ASTIN Bulletin 35, 45-60.

[8] Drekic, S., Dickson, D.C.M., Stanford, D.A. and Willmot, G.E. (2004) On the distribution of the deficit at ruin when claims are phase-type. Scandinavian Actuarial Journal 2004, 105-120.

[9] Garcia, J.M.A. (2005) Explicit solutions for survival probabilities in the classical risk model. ASTIN Bulletin 35, 113-130.

[10] Gerber, H.U. (1979) An Introduction to Mathematical Risk Theory. Philadelphia, PA, S.S. Huebner Foundation.

[11] Gerber, H.U., M.J. Goovaerts and R. Kaas (1987). On the probability and severity of ruin. ASTIN Bulletin 17, 151-163.

[12] Li, J., Dickson, D.C.M. and Li, S. (2015) Some ruin problems for the MAP risk model. Insurance: Mathematics \& Economics 65, 1-8. 
[13] Prabhu, N.U. (1961) On the ruin problem of collective risk theory. Annals of Mathematical Statistics 32, 757-764.

[14] Ren, J. (2008) On the Laplace transform of the aggregate discounted claims with Markovian arrivals. North American Actuarial Journal 12(2), 198-206.

[15] Willmot, G.E. (2007) On the discounted penalty function in the renewal risk model with general interclaim times. Insurance: Mathematics \& Economics 41, 17-31.

[16] Willmot, G.E. (2015) On a partial differential equation of Seal's type. Insurance: Mathematics \& Economics 62, 54-61. 


\section{University Library}

\section{- M M I N E R VA A gateway to Melbourne's research publications}

Minerva Access is the Institutional Repository of The University of Melbourne

Author/s:

Dickson, DCM

Title:

A note on some joint distribution functions involving the time of ruin

Date:

2016

Citation:

Dickson, D. C. M. (2016). A note on some joint distribution functions involving the time of ruin. Insurance: Mathematics and Economics, 67 (2016), pp.120-124. https:// doi.org/10.1016/j.insmatheco.2015.12.005.

Persistent Link:

http://hdl.handle.net/11343/120632 